\title{
Un mismo continente, dos Américas: viajeros latinoamericanos en los Estados Unidos, siglo XIX*
}

Carlos Sanhueza**

Resumo: Este artigo trata das experiências de viajantes latino-americanos nos Estados Unidos durante o século XIX. Durante este período, estes viajantes que pareciam ter uma posição reservada frente à cultura anglo-saxônica, relacionaram-se com valores tais como espiritismo, afeição e solidariedade, ao contrário dos valores que acreditavam ser os indicadores da cultura norteamericana, como utilitarismo, materialismo e grosseria. Considerando esta atitude, este artigo discute de que maneira o conceito da identidade latinoamericana emerge como resultado de um encontro entre fronteiras culturais que separa estas duas Américas.

Abstract: This article deals with the experience of Latin American travelers in the United States during the 19th century. During this period, these travelers appeared to have a defensive position towards Anglo-Saxon culture, associating themselves with such values as spiritualism, affection and solidarity, in contrast with those values they believed were indicative of North American culture, such as utilitarianism, materialism and bluntness. It is taking into account this attitude that this article discusses how the concept of Latin American identity emerged as a result of the clash between the cultural boundaries which separate these two Americas.

Palavras-chave: Viajantes latino-americanos. Imagem dos Estados Unidos. Identidade latino-americana.

Key words: Latin American travelers. Image of the United States. Latin American identity.

* Este artículo forma parte de un proyecto de investigación FONDECYT-Regular $\mathrm{N}^{\mathrm{o}} 1070453$ referido a viajeros latinoamericanos en los Estados Unidos durante el siglo XIX. Agradezco a la Comisión Nacional de Investigación Científica y Tecnológica de Chile (CONICYT) el apoyo prestado. Así mismo deseo reconocer el infatigable trabajo prestado por Pamela Maturana.

** Universidad de Talca/Chile; e-mail: casanhueza@utalca.cl

Estudos Ibero-Americanos, Porto Alegre, v. 35, n. 1, p. 73-93, jan./jun. 2009 
El viaje de latinoamericanos a los Estados Unidos, en especial de su élite intelectual, es posible de encontrarlo desde antes de la centuria decimonónica. Ya desde fines del siglo XVIII un importante flujo de latinoamericanos se sintió atraído por recorrer y escribir sobre Norteamérica, principalmente aquellos involucrados en el proceso de Emancipación de España, como Francisco de Miranda, Simón Bolívar o José Miguel Carrera. Destacable resulta como en ellos la búsqueda de una independencia del dominio imperial europeo hizo que se sintieran vinculados al movimiento norteamericano independentista en la noción ilustrada de la liberación y autodeterminación de los pueblos. Lo anterior explica por qué dichos viajeros, en su mayoría, no indagaron aquello que los diferenciaba del País del Norte, ni asumieron un distanciamiento crítico. ${ }^{1}$ Fue desde mediados del siglo XIX, una vez que la separación de España se hubo concretado, cuando el desplazamiento a los Estados Unidos adquirió un nuevo sentido. El presente trabajo analiza aquellos elementos históricos involucrados en esta fractura post-emancipación, enfatizando cómo, a partir de las posiciones tomadas por los viajeros al otro lado del Río Grande, se concibió la existencia de dos tipos de Américas. Su objetivo es analizar en qué sentido la ficción de un subcontinente latinoamericano apareció en las posiciones de los viajeros como una defensa de lo propio y exaltación de unos valores asociados, frente a lo que percibían como característico del país del norte.

El estudio de la experiencia de latinoamericanos en los Estados Unidos durante el siglo XIX ha girado en torno a dos ejes temáticos. Una vertiente se ha detenido en la presencia de inmigrantes latinoamericanos principalmente en la llamada "fiebre del oro" en la región de California, en textos como los de Roberto Hernández, Los chilenos en San Francisco de California (1930); Edwin Beilharz y Carlos López, eds., We Were 49ers! Chilean Accounts of the California Gold Rush (1976) y Gilberto Harris, Emigración y politicas gubernamentales en Chile durante el siglo XIX (1996). Dichos trabajos más bien han destacado la vida de tales inmigrantes: los conflictos y dificultades sufridas en tanto extranjeros. La segunda perspectiva ha tomado a los viajeros como tales. Sin embargo, estos últimos frecuentemente aparecen circunscritos a introducciones o prefacios en nuevas ediciones de relaciones de viaje como los referidos a José Martí, Domingo Faustino Sarmiento o José Arnaldo Márquez. También se ha incluido el estudio de viajeros al abordar las percepciones que América Latina ha ido construyendo de

1 Respecto de los viajeros independentistas en los Estados Unidos ver RACINE, 2003. 
los Estados Unidos, como en Carlos Rama La imagen de los Estados Unidos en la América Latina (1975). Finalmente es posible advertir una posición que analiza, desde un punto de vista literario, un conjunto de viajeros de un país determinado a partir de sus descripciones los Estados Unidos, como en Lilianet Brintrup, Viaje y escritura. Viajeros Románticos chilenos (1992); Beatriz Colombi, Viaje intelectual. Migraciones y desplazamientos en América Latina (1880-1915), (2004) y David Viñas, Viajeros argentinos a Estados Unidos (2008) o de casos particulares, como respecto de Domingo Faustino Sarmiento, Ramón de la Sagra; Justo Sierra O`Reilly o Eduarda Mansilla. ${ }^{2}$

En muchos de los trabajos anteriormente mencionados, por lo general, se ha privilegiado el corpus de los relatos de viajes como una fuente que describe una situación individual, o nacional, pero siempre limitando su influencia en un espacio específico y acotado. Sin embargo, hasta ahora, prácticamente no se ha estudiado el papel jugado por dichos viajes en la concepción latinoamericana del Nuevo Mundo posterior a la Emancipación. Este artículo busca aportar en tal sentido, analizando como, a pesar de los diferentes enfoques adoptados por los viajeros, dichos desplazamientos marcaron un hito, unos vínculos que, de una u otra forma dejó sus huellas sobre el diálogo entre la América Sajona y la Latina.

\section{La identidad latinoamericana desde el otro lado del Río Grande}

François-Xavier Guerra (2003) ha destacado que la identidad latinoamericana es una construcción cultural, entendida ésta de manera amplia, sin limitarla tan sólo a elementos como la lengua, la religión, los mitos históricos o las particularidades étnicas. La identidad de la porción hispana del Nuevo Mundo, en este sentido, se representa sobre la base de una territorialidad que no siempre corresponde a una geografía real: América Latina como subcontinente se concibe sólo a condición de una separación con respecto a otra América. De ahí que la división entre una América sajona y una latina ha sido vista desde la conformación de fronteras culturales antes que netamente políticas (Michaelsen; Johnson, 2003). Tal y como en otros espacios geoculturales donde se han configurado grandes divisiones del mundo - oriente y occidente; norte y sur - así en América, el Río Grande (como el Rhin entre Alemania

2 Ver al respecto HERNÁNDEZ, 1930; BEILHARZ/LÓPEZ, 1976; HARRIS, 1996; RAMA, 1975; BRINTRUP, 1992; COLOMBI, 2004 y VIÑAS, 2008. 
y Francia) ha trazado la cesura entre dos mundos que se advierten disímiles, cuando no opuestos (Sullivan, 2005, p. 3-24).

Walter Mignolo (2000 y 2005) afirma que esta división entre ambas Américas corresponde a una elaboración europea ocurrida en el siglo XVIII. Si bien América desde su incorporación a la visión tripartita del mundo (Europa, África y Asia) fue anexada como una totalidad al discurso occidentalista - una suerte de extensión de Europa - el nuevo orden colonial que emergió en la llamada segunda fase de la modernidad según Immanuel Wallerstein, o segunda fase de una globalización acelerada al decir de Ottmar Ette, marginó a la porción hispana de tal unidad geocultural. ${ }^{3}$ El orden colonial moderno se construyó sobre la base de la supremacía de un conjunto de naciones (Inglaterra, Francia, Alemania) que desplazaron a las antiguas potencias imperiales vinculadas al sur europeo (España, Portugal). Occidente se definió privilegiando lo sajón, lo germano, lo franco (con todos sus elementos lingüísticos, religiosos y culturales asociados), ubicando al resto del mundo en una posición de subalternidad. ${ }^{4}$ Por lo anterior se incluyó a la América sajona en los discursos de la civilización occidental y se marginó a la América hispana, tal y como había ocurrido en Europa con la propia España. Esta fractura del Siglo de las Luces se advierte con la llegada del orden postcolonial americano y la conformación de los estados nacionales en el siglo XIX. De ahí que, tal y como lo destaca Walter Mignolo (2000), ambas Américas perciban diferentes la separación de las metrópolis europeas: mientras Simón Bolívar se preocupó de la indiferencia de Europa (o sea, Europa del norte) en relación al proceso independentista del Nuevo Mundo, Thomas Jefferson advierte un horizonte en el cual sólo aparece América y los americanos. Jefferson buscaba una América para sí misma pues él se definía como parte del mundo occidental moderno; Bolívar hacía un esfuerzo por integrar a la porción hispana del Nuevo Mundo a la modernidad.

Por otro lado el propio papel de los Estados Unidos como potencia continental, se percibía diferente a uno y otro lado del Río Grande. En otras palabras: a medida que los Estados Unidos fortalecía su presencia tendía a justificar su supremacía en tanto se percibía heredero de la civilización que España había perdido. Sin embargo, aquello que para el País del Norte significaba una expansión natural a partir de su entrada a la modernidad, comenzaba a presentarse para las élites latinoamericanas

3 WALLERSTEIN, 1974-1989; ETTE, 2002. En relación al discurso occidentalista de los viajeros latinoamericanos en Europa ver SANHUEZA, 2007, p. 51-75.

4 VENN, 2000; CHAKRABARTY, 2000 y 2002); BURUMA/y Avishai MARGALIT, 2004. 
como una amenaza. E incluso, no pocos veían el poder hegemónico norteamericano definitivamente como un riesgo para las conquistas que se habían logrado con la Emancipación. Junto a esta desconfianza ante los Estados Unidos se advertía una actitud de interés de parte de los latinoamericanos respecto de un modelo republicano que, sin desarrollarse en suelo europeo, había incluso superado al Viejo Continente en participación y expresión de una soberanía popular. Es justamente en este cuadro contradictorio de desconfianza y admiración; desdén e imitación, donde se inserta el desplazamiento de latinoamericanos allende el Río Grande. De manera creciente, en particular desde mediados de la centuria decimonónica, un conjunto de intelectuales, políticos, comerciantes, escritores, aventureros y mujeres venidos de América Latina alcanzaron tierras norteamericanas. Este interés no sólo respondió a un afán literario, mercantil, de exilio político o de turismo: los viajeros buscaron entender y a su vez explicarse las diferencias entre dos espacios de colonización europea disímiles. ¿Dónde radicaba la distancia que separaba y a la vez conformaba una frontera entre ambas Américas? ¿Cómo poder explicarla? ¿Era posible tomar a los Estados Unidos como un punto de referencia para la construcción de las nuevas repúblicas hispanoamericanas?

\section{Un viaje al futuro}

Domingo Faustino Sarmiento, en su primer viaje a los Estados Unidos en 1847 una vez concluido el periplo europeo, no podía dejar de comparar y admirarse ante lo que percibía en aquellas tierras. El intelectual argentino ve alzarse a los Estados Unidos como la superación de todo aquello que le había incomodado en el Viejo Mundo y respecto de lo que aborrecía de la América Hispana. Los Estados Unidos eran percibidos como un pueblo independiente y libre como el aire, sin tutores, sin ejército, $i$ sin bastillas. Un lugar que no poseía reyes, ni nobles, ni clases privilegiadas, ni hombres nacidos para mandar, ni máquinas humanas nacidas para obedecer (Sarmiento, 1997, p. 297-298). Se ha seguido la grafía original). Estas características del pueblo norteamericano no constituían, en ningún modo, abstracciones o juicios políticos: antes bien, el viajero las veía materializarse en el acontecer cotidiano de la aldea, una suerte de microcosmos del estilo de vida norteamericano. ${ }^{5}$ En efecto, Sarmiento veía a la aldea como el centro

5 Respecto a la noción en Sarmiento de la aldea como microcosmos he seguido a ERRÁZURIZ, 2008, p. 40-63. Ver además KATRA, 1994, p. 73-100 y 1997, 853-912. 
de la vida política norteamericana, espacio que contenía al país con todos sus accidentes, cosa que no puede decirse de nación alguna. Según Sarmiento, la aldea en los Estados Unidos, a diferencia de Chile o Francia donde ésta era la negación de su ser nacional, concentraba ya todo el estado, en su gobierno civil, su prensa, sus escuelas, sus bancos, su municipalidad, su censo, su espíritu i su apariencia (Sarmiento, 1997, p. 298). De tal forma que la igualdad no se quedaba en meros discursos: antes bien, se concretaba desde el hogar mismo. Justamente la vista directa de la aldea le permitía al literato rioplatense, más allá de la teoría, asir cotidianamente su vida republicana. De esta manera era posible advertir en qué medida la institución republicana era el origen del progreso de la población norte-americana, en tanto ésta encerraba en sí las fuerzas productoras i de enerjía física $i$ moral de un pueblo avezado a las prácticas de la libertad, del trabajo i de la asociación (Sarmiento, 1997, p. 335-337).

Ahora bien, la existencia de un régimen republicano no explicaba por sí mismo la grandeza de los Estados Unidos. ¿Dónde radicaba, en definitiva, el éxito de dicha porción del Nuevo Mundo? Para el viajero argentino la cesura que distanciaba al país del norte del resto del mundo no era la libertad de cultos: para cada secta religiosa las otras son como si no existieran i por tanto la libertad es nula en sus efectos para cada una separadamente (Sarmiento, 1997, p. 315). Tampoco se entendía el fenómeno norteamericano atendiendo las facilidades que ofrece un país nuevo, con terrenos vírgenes y de fácil adquisición, tal y como argumentaban los europeos. A dicha sentencia, Sarmiento le oponía el caso sudamericano, pródigo en tierras inexploradas y que, sin embargo, conformaba un territorio de atraso, pobreza e ignorancia. Menos aún se podía encontrar una respuesta en el elemento étnico. Para Sarmiento (1997, p. 315),

[...] la aptitud de la raza sajona no es tampoco explicación de la causa del gran desenvolvimiento norte-americano. Ingleses son los habitantes de ambas riberas del río Niágara, i sin embargo, el ojo percibe que son dos pueblos distintos.

De modo que no era la libertad en un sentido abstracto, ni en tanto reconocimiento político, sino más bien su uso lo que en definitiva explicaba las diferencias de los Estados Unidos con respecto a Europa y a América Hispana. La sola existencia de libertades no aseguraba el progreso: en Sarmiento (1997, p. 315) lo importante era observar la manera como ésta obraba para producir los prodigios de prosperidad 
que los Estados-Unidos ostentan. He aquí la trascendencia del periplo norteamericano en el escritor argentino en la medida en que, desde la cotidianidad, el monótono devenir, captaba la libertad actuando sigilosamente. En este sentido, sólo un viajero atento a lo micro podía profundizar, darse cuenta de los efectos de los principios republicanos en la vida misma de sus habitantes. De allí, también, la justificación de sus descripciones: Fijome en estos detalles porque ellos solos bastan a caracterizar a un pueblo i suscitan un cúmulo de reflexiones (Sarmiento, 1997, p. 297).

José Martí se radicó en los Estados Unidos entre los años 1880 y 1894. El escritor cubano, en rigor más que viajero un exiliado, al igual que Sarmiento escudriña respecto de aquellos argumentos que dan cuenta de la existencia de dos Américas. La misma libertad que destacaba Sarmiento, según Martí, era la que posibilitaba el avance en el País del Norte. Es justamente tal espacio de emancipación el que había empujado a millones hacia estas tierras del Nuevo Mundo. En palabras de Martí (2003, p.137): He aquí el secreto de la prosperidad de los Estados Unidos: han abierto los brazos. Esta apertura, empero, no significaba tan sólo el acceso a tierras, a bienes materiales. Si así fuera no se podría explicar porqué se elegía la porción sajona y no la hispana del Nuevo Mundo, más si se considera que en ambos espacios americanos se podían iniciar empresas de colonización en terrenos deshabitados. Lo que, bajo el punto de vista de Martí, distinguía a los Estados Unidos era su igualdad: el hecho de que allí no se discriminase por causas políticas u orientaciones religiosas, como si ocurría en la América Hispana y en Europa. En definitiva, el espacio de libertad que ofrecía el País del Norte era el origen de su grandeza, como la explicación de su atracción en los millones que acudían dichas tierras. Según Martí (2003, p. 137), Luchan los hombres por pan y por derecho, que es otro género de pan; y aquí [en los Estados Unidos] hallan uno y otro..."

En términos similares a los de Martí, el publicista y político colombiano Salvador Camacho Roldán (1973, Tomo I, p. 175) trata de explicarse las causas de la prosperidad excepcional de los Estados Unidos. El viajero, haciendo un balance de su periplo norteamericano en 1887, afirma que son las instituciones republicanas, aceptadas sin contradicción por todos los habitantes, la agencia inicial de esa transformación. Según el viajero colombiano, mientras en el continente europeo, ardía la guerra entre las naciones más civilizadas, en los Estados Unidos progresaban la libertad religiosa, la libre imprenta, la libertad industrial, el comercio sin trabas. He ahí la diferencia con la América al otro lado del Río Grande: 
No tuvieron las Repúblicas hispano-americanas [...] todas estas ventajas. Adoptaron las instituciones republicanas, pero carecían de las tradiciones antiguas de libertades religiosas implantadas desde su origen en las colonias inglesas [...]. La intolerancia religiosa, principio esencial del gobierno español, había dejado raíces profundas; [...] subsistieron [...] gran parte de los monopolios de origen colonial; sus libertadores mismos, en fin, fueron los primeros jefes de la reacción de su propia obra... (Camacho, 1973, p. 175, Tomo I).

Para Camacho Roldán no eran en sí las instituciones las que daban cuenta de la prosperidad del País del Norte, sino más bien su tradición y asimilación por parte de la población. En este balance el pasado colonial se erguía como un factor explicativo de primer orden.

\section{Un viaje al corazón del materialismo}

No siempre las observaciones de los viajeros latinoamericanos al otro lado del Río Grande, si bien mostraban la gran distancia que existía entre las dos porciones del Nuevo Mundo, giraban en torno al halago y la admiración. En otros términos: a pesar del evidente adelanto material de los Estados Unidos, no pocos viajeros destacaron los claroscuros de tales logros. El propio Martí, junto con asombrarse ante la urbe norteamericana, fustigaba sus efectos sobre la vida de sus habitantes. La rapidez, la vertiginosidad, le hacía revalorar lo que había dejando al otro lado del mundo:

La vida en Venecia es un góndola; en París, un carruaje dorado; en Madrid un ramo de flores; en New York, una locomotora de penacho humeante y entrañas encendidas. Ni paz, ni entreacto, ni reposo, ni sueño. La mente, aturdida, continúa su labor en las horas de noche dentro del cráneo iluminado. Se siente en las fauces polvo; en la mente, trastorno; en el corazón, anhelo. Aquella calma conventual de las ciudades de la América del Sur, donde aún con dedos burdos pasa las cuentas de su rosario, desde su ermita empinada, el Padre Pedro, - en esta tierra es vida. Se vive a caballo en una rueda. Se duerme sobre una rueda ardiente. Aquí los hombres no mueren, sino que se derrumban: no son organismos que se desgastan, sino Ícaros que caen. ${ }^{6}$

Los viajeros latinoamericanos constaban que no sólo se estaba ante la presencia de un ritmo distinto, lo que hablaría más bien de una vida pueblerina en América Hispana al comparársela con la Sajona, sino

6 MARTÍ, 2003, p. 277. Cita tomada también por Colombi, 2004, p. 48. 
que además en medio de un mundo construido sobre otras prioridades y escalas de valor a las utilizadas al sur del Río Grande. El chileno Benjamín Vicuña Mackenna advierte tales disparidades entre los Estados Unidos y la América Hispana desde su periplo norteamericano en 1853.

A fines de 1852, con poco más de veinte años de edad, llega Vicuña Mackenna a los Estados Unidos. El motivo de su estadía era el destierro político, pero también la idea de un recorrido formativo que lo dotase de experiencias, conocimientos y hasta de estudios. Inquieto y buscador, en cerca de siete meses recorrió la región de California, San Francisco, Nueva Orleáns, Ohio, Nueva York, Boston, Washington, aparte de pequeños poblados. Allí reunió información, asistió a espectáculos, recorrió cementerios, plazas, plantaciones, edificios públicos y de gobierno, áreas productivas y bibliotecas. Todo lo registraba, todo lo comparaba. Pero en especial intentaba describir al estadounidense, al yankee. Vicuña Mackenna no sólo deja sentado, desde sus observaciones de la vida cotidiana en los Estados Unidos, aquellos aspectos admirados en los Estados Unidos y que tanto asombro causaban a los viajeros venidos del sur del continente, sino también una suerte de carácter que definía al americano del norte respecto de su vecino austral.

Para el viajero chileno la cultura de los Estados Unidos giraba en torno a la forma más rápida de conseguir dinero. El mentado time is money, en definitiva, significaba el acumular la mayor cantidad de dinero en el menor tiempo posible. Sólo así se podía entender el hecho de que los norteamericanos buscasen una y otra vez el engaño, la artimaña, la trampa que les permitiese alcanzar tales fines materiales. Al respecto Vicuña Mackenna logra plasmar este afán en una palabra: humbug. Dicha palabra la utiliza el viajero como una llave maestra que le permite desentrañar el espíritu de todo un pueblo:

El humbug es una palabra que aunque el diccionario traduce en español como 'engaño, dolo, impostura y trampa', es, sin embargo, intraducible en su familiar sentido americano; es un yankesismo único en su especie, como el go a head! Y el money make lo son en otro sentido. Sólo el que haya residido en los Estados Unidos puede valorizar esta frase que, como ciertas plantas peculiares, no puede aclimatarse en parte alguna si se le arranca al suelo que ha nacido. El humbug es omnímodo, es universal, cosmopolita, en todo el vasto territorio de la unión. Hay hombres humbugs, cosas humbugs, animales humbugs, ideas humbugs. Un presidente americano que, como Taylor, traiciona a su partido en el poder, es un humbug. Van Buren, que produce una crisis monetaria en vez de enriquecer al país, es un humbug. (Vicuña Mackenna, 1856, p. 43) 
Una y otra vez el viajero chileno traduce culturalmente las formas de vida norteamericana sobre la base de la palabra inglesa humbug. A partir de esta posición crítica, Vicuña Mackenna logra develar los claroscuros del progreso en el país del Norte: materialismo, competencia feroz, fijación por el dinero. En este ambiente la vida humana perdía todo valor, todo sentido. El individualismo, en este aspecto, no implicaba tan sólo el fortalecimiento de la ciudadanía, sino también de un egoísmo extremo. Lo anterior no surgía de un conjunto de prejuicios o miradas nostálgicas sobre lo que se había dejado atrás: la experiencia de viaje misma, el observar la vida cotidiana, aunque fuesen pequeños hechos, se lo demostraba a cada paso:

En la mitad de nuestro viaje sentí un pequeño sacudón y el tren se detuvo un instante. Curtis [un norteamericano que lo acompañaba] indagó la causa y me dijo no era nada!...Era simplemente un hombre que, estando dormido, atravesado en los rieles, había sido dividido en dos mitades por la locomotora! Este nada! que estaba escrito en los semblantes de todos, que conversaban y reían como antes, me aterró; pero, después vi realmente que aquello era nada en los Estados Unidos, donde sólo causan una ligera impresión las whole sale butcheries, o las matanzas al por mayor. (Vicuña Mackenna, 1856, p. 52. Destacado del original).

Aterrador no era sólo el darse cuenta lo poco que la vida humana importaba en un país volcado a la satisfacción personal: lo que remecía al viajero era observar cómo aquello se asumía como algo natural. ¿Podía llegar a ser un modelo de formación republicana semejante actitud del ciudadano común y corriente? ¿Podía tomarse al País del Norte, en definitiva, como un punto de referencia para las nuevas repúblicas hispanoamericanas? Al concluir el periplo norteamericano, Vicuña Mackenna, decepcionado por los Estados Unidos, remarca lo que según él - en definitiva - diferenciaba a ambas Américas:

A fines de julio de 1853 me preparaba para dejar los Estados Unidos. Había yo penetrado en aquel país, henchido de un simpático y ardiente optimismo; salía de él con el desengaño en el corazón. Mi afección por su pueblo, mis ilusiones por su grandeza social, todo se había desprendido de mí, paso a paso, sepultado en mi camino por la inmensa mole de materialismo que hunde a este país, al paso que le sirve de pedestal; yo llevaba conmigo sólo mi admiración por su poder, pero debo confesarlo, admiración desnuda, sin simpatía ni entusiasmo, como la que inspira el océano sin fin o el huracán que nos amenaza...Mi admiración, en efecto, por los Estados Unidos, era como a pesar mío, pero ¿quién pudiera a reconocer los inmensos 
destinos que aguardan a este pueblo, la primera nación del mundo hoy mismo, en el sentido en que el mundo comprende el poder y la grandeza? ${ }^{7}$

La experiencia de viaje en los Estados Unidos le había dejado a Vicuña Mackenna un sabor amargo, un desengaño en el corazón. Nada se obtenía de una grandeza social si ésta se construía sobre la base de un materialismo ilimitado, conductas inhumanas (como el descrito en la escena del tren), impersonalismos. Pero lo que era aún peor, y que en algún sentido explican este sabor amargo, era constatar que justamente tales conductas irreprochables le servían al País del Norte de pedestal. Aquí se instalaba una paradoja: el viajero sudamericano llegaba con la expectativa de encontrarse con el progreso, sin embargo, constataba al final de su periplo que dicho sitial sólo se alcanzaba sacrificando lo espiritual, lo verdaderamente humano. Esta paradoja devenía en tragedia una vez que se advertía que, en definitiva, era éste el verdadero camino a la modernidad. Esto explica por qué el viajero, a pesar suyo y de sus críticas, termine admirando al País del Norte.

Vicuña Mackenna se percata que el significado a partir del cual el mundo comprende el poder y la grandeza estaba del otro lado del Río Grande. Dicha constatación le permitía visualizar las fronteras que separaban a las dos Américas. ¿Se estaba ante la presencia de dos modelos antagónicos de civilización?:

La historia (...) ha desarrollado en la humanidad dos clases de civilización, la una de la moral y de la inteligencia, y la otra del materialismo, mejor conocido por el sistema utilitario. Aquella, de que la raza latina fue la madre y la nodriza (...) ha quedado (...) depositada entre nosotros (...). ¿Poseen ambos elementos de grandeza los Estados Unidos? ¿Sirve la inteligencia al perfeccionamiento de la sociedad, a la depuración del error, al ensanche de los dominios de la razón, de la justicia y de la libertad? No, por cierto! La moral (...) se mantiene solamente (...) en el rincón doméstico (...). La civilización intelectual se ha hecho más esparcida, pero como la luz que se derrama en el vacio se ha debilitado por falta de un foco común y de pábulo diario. La civilización material sí es portentosa, inconcebible, inaudita aquí (Vicuña Mackenna, 1856, p. 100. Destacado del original.) $^{8}$

7 Ibid, p. 99. Esta crítica al materialismo norteamericano la volverá a hacer en VICUÑA MACKENNA, 1867, p. 193-200, Tomo I. E incluso en este último libro cita su propio diario de viajes para confirmar su visión de los Estados Unidos. Respecto de la misión secreta de Vicuña Mackenna en los EEUU ver Cristián Guerrero Yoachim, 1986, p. 35-86.

8 VICUÑA MACKENNA, 1856, p. 100. Destacado del original. 
La civilización material se erigía como soberana en los Estados Unidos. El viajero reconoce que el País del Norte había desarrollado como ningún otro pueblo la libertad y los derechos del hombre. Sin embargo, la inclinación mercantilista de esta raza sajona había terminado por abusar de ese noble poder, lo ha conquistado para sí y lo deja arrebatar a los demás (Vicuña Mackenna, 1856, p. 247). Vicuña Mackenna percibía en qué sentido la noción de modernidad, progreso y occidentalidad comenzaba a redefinirse en el mundo desde los Estados Unidos y a partir de una clara impronta materialista e inhumana. Al despedirse de los Estados Unidos, Vicuña Mackenna se pregunta: ¿Qué podemos esperar de aquella nación?:

Digámoslo al fin como nuestra despedida de sudamericano al suelo de la Unión del Norte, la América del Sur nada tiene que esperar de la del norte. Podría recibir mucha de aquella, pero esos bienes de civilización no nos serían ofrecidos como un don ni aceptados tampoco. Además están puestos en venta en todas partes y no hay más que tener dinero para comprarlos. En verdad, si la América del Norte comunicara algo de su ser y de su influencia a país alguno, no sería ciertamente por expansión generosa, sino, cuando más, por una egoísta y fría asimilación de intereses (Vicuña Mackenna, 1856, p. 107).

Vicente Pérez Rosales, de manera aún más próxima que Benjamín Vicuña Mackenna, observó el lado inhumano del materialismo norteamericano en medio de la fiebre del oro. Por 1848 el viajero chileno, después de una serie de fracasos económicos una vez vuelto a Chile desde su estadía parisina, decide ir a probar suerte con el oro de California. Junto a un grupo de compatriotas de diferentes estratos sociales y procedencias, llegó a los Estados Unidos a fin de hacer realidad el sueño de la riqueza minera. ${ }^{9}$

Los aventureros chilenos arribaron primero a San Francisco, llegando más tarde hasta Sacramento. Allí se desarma rápidamente la idea de un enriquecimiento fácil, pues se ven enfrentados a la realidad de un lugar entre hombres que, al decir del propio Pérez Rosales, no tenían más Dios que el oro, más derecho que el del más fuerte, ni más Corte de Apelaciones que la de las armas.

9 El texto que relata tales travesías es Diario de un viaje a California (1848-1849) que en su primera edición fue publicada en tres partes bajo el título: "Algo sobre California" en Revista de Santiago, Primera Época, T. 6, 3-10, 127-136, 185-193, 1850. Este viaje además fue incorporado en PEREZ ROSALES, 1886. 
Allí Pérez Rosales conoce la faz oscura del apogeo norteamericano, como también las complejas y hasta crueles condiciones de la fiebre del oro californiana. Después de no haber podido con la competencia y la hostilidad del lugar, habiendo intentado invertir sin éxito en la zona, se vio en la obligación de volver al país, casi arruinado. ${ }^{10}$

Destacable resulta la posición de Pérez Rosales en medio de los Estados Unidos. Una y otra vez, a lo largo de su relato, critica la barbarie del yanqui: criaturas grotescas, sin modales, ni fineza. Pero, más allá de tales comentarios propios de quien se sabe perteneciente a la alta sociedad de su país de origen, están aquellas observaciones referidas al norteamericano en tanto ciudadano, como también a su sistema judicial. E incluso, el sentido de autoridad, tan alabado por otros viajeros en los Estados Unidos, es visto - en la realidad de la vida diaria en California - desde otra perspectiva:

La suprema autoridad de San Franciscano es un alcalde como dicen muchos, es un yanqui más o menos borracho a quien llaman alcalde y que sólo sirve, si es entre dos yanquis, de juez conciliador, si es entre un yanqui y uno que hable español, para hacer que el español sea reo y pague las costas del proceso [...] (Pérez Rosales, 2007, p. 89).

A diferencia de otros viajeros hispanoamericanos, como Domingo Faustino Sarmiento, Pérez Rosales se inserta en medio de un territorio fronterizo. Es a partir de allí donde éste observa en qué sentido la violencia conformaba un código de conducta aceptado, así como el atropello al más débil algo cotidiano y evidente. Sin embargo, a pesar de haber sufrido en carne propia la marginación y el racismo, pareciera que aceptase tales males en tanto parte del desarrollo y la inserción en el mundo moderno. Es como si, algo similar a lo experimentado por Benjamín Vicuña Mackenna, su propia experiencia traumática le hubiese mostrado que no era posible alcanzar la modernidad sin un alto costo. Sólo tendiendo en cuenta lo anterior se pude comprender el tono del apéndice Algo sobre California al finalizar su diario de viajes. Si bien dicho texto lo incluye con el propósito de dejar establecido "...hasta que punto su desarrollo [de los EEUU] y engrandecimiento inevitables pueden afectar los intereses y el futuro bienestar de las repúblicas occidentales", va más allá (Pérez Rosales, 2007, p. 123). A fin de poder explicarse el origen de la prosperidad y el espíritu emprendedor de los

${ }^{10}$ Respecto de la experiencia de los chilenos en la fiebre del oro californiana de 1849 ver ROHRBOUGH, 1997, p. 224-226. Ver además LÓPEZ, 1975. 
Estados Unidos, Pérez Rosales trata de encontrar aquello que le permite a la porción sajona de América un engrandecimiento inevitable respecto de homónima hispánica, sumida en el atraso y el abandono.

En primer lugar, el viajero chileno parte de la premisa que no es la riqueza minera en sí misma lo que explica la prosperidad de California:

No es pues el oro muy agotable en sus minas el primer elemento que ofrece a California un venturoso porvenir. El genio anglobretón, el espíritu de asociación, de mejoras, de empresas, miras extensas, tolerancia religiosa, actividad que en vez de mortificar irrita, constancia que raya en tenacidad, y firme persuasión que la enérgica voluntad unida a los recursos del siglo diecinueve todo lo vence, he allí las bases harto más sólidas que el eventual recurso del oro en las que se apoya su futuro engrandecimiento (Pérez Rosales, 2007, p. 124).

De modo que no se trataba de un porvenir cimentado en una frágil riqueza momentánea que en algún momento iría a acabarse. De ser así de nada serviría observar lo acontecido en California: bastaría tan sólo volcarse al subsuelo latinoamericano y explotarlo, tal y como había ocurrido en la América Hispana desde la conquista española. La suma de un conjunto de factores (genio anglo-bretón, espíritu de asociación, tolerancia religiosa, constancia, voluntad) era lo que finalmente se unía al oro, a los recursos del siglo diecinueve, pavimentando el camino de prosperidad y engrandecimiento.

En otro sentido, la experiencia de viaje en la región californiana conformaba una suerte de "laboratorio": una oportunidad inigualable de comprobación de su tesis desarrollista al comparar los cambios allí acontecidos tras su anexión a los Estados Unidos. Reflexionar sobre tales modificaciones le permitía al viajero encontrar las causas de la prosperidad californiana más allá de los destellos reflejados por la fiebre del oro. De allí que Pérez Rosales busque poner a uno y otro lado de la balanza lo que había sido California bajo el régimen español, por el espacio de más de doscientos años, al comparar este dilatado período de sueño y de letargo, con sólo dos años de vida bajo sus nuevos posesores. $^{11}$

Un elemento clave en dicha comparación lo constituía el cambio acontecido en la zona en términos de su estructura urbana, infraestructura

${ }^{11}$ Ibid. Esta comparación entre la administración norteamericana y mexicana de California también la incluye en sus memorias. Ver PÉREZ ROSALES, 1886, p. 218-223. 
y apertura al comercio. En su Apéndice destaca Pérez Rosales que, mientras la administración hispana convirtió los puntos más importantes de sus territorios en misiones, el régimen estadounidense construyó ciudades y ciudadanía: las urbes prontamente adquirieron una forma más regular; los montes resonaron en todas direcciones bajo la hacina industriosa del yanqui; una multitud de calles nuevas y de hermosos edificios dieron otro ser a la ciudad. El viajero sudamericano remarcaba en qué sentido los estadounidenses introdujeron aduanas, fortalezas, escuelas, casas de depósitos; trajeron ingenieros, levantaron planos topográficos. Junto a lo anterior, Pérez Rosales (2007, p. 128-129) destaca cómo los nuevos dueños de California no le exigieron a los recién llegados ni las credenciales de su procedencia, ni su adoración religiosa.

En el fondo, a decir del viajero sudamericano, lo que habían hecho los norteamericanos era la propagación de las luces. Sólo de esta manera era posible comprender como, en un espacio brevísimo de tiempo, la nación del Norte había logrado vencer el atraso de la raza española. Observar tales cambios permitía, por un lado, abrigar la esperanza de un progreso en la América Hispana. Sin embargo, por otro lado, tal constatación dejaba en evidencia que, en definitiva, el obstáculo para la América Latina era la hispanidad misma: tras el periplo norteamericano se hacía evidente para el viajero chileno que el mal está en la masa de la sangre. En este punto, las dos Américas se separaban inexorablemente. El juicio del viajero era concluyente:

Triste es decirlo, pero hay en la raza hispanoamericana un fondo de inactividad, de propia desconfianza, de insocial egoísmo, que rechaza el concurso de los brazos y de los capitales; un espíritu de esperarlo todo el tiempo y de considerar prematura toda clase de reforma con tal que sea fundamental; y sobre todo, aquella desgraciada manía de fijarse con ahínco en las causas eventuales que pueden hacer fracasar una empresa, y no acometer por esto las más provechosas como si hubiese algún cálculo humano que no esté sujeto al inexorable puede suceder (Pérez Rosales, 2007, p. 127. Destacado del original).

Nada se obtenía siguiendo los pasos que los norteamericanos habían dado - migraciones europeas, apertura al comercio, transformaciones urbanas -, sino se combatía aquel fondo de inactividad, de propia desconfianza y de pasividad heredados de España. El viajero chileno, a fin de cuentas, se percataba que la entrada a la modernidad, a un 
occidente cada más representado por el mundo anglosajón, suponía algo más que la introducción de maquinarias, de nuevos métodos educativos o de constituciones políticas. El costo era alto, tal y como lo habían percibido en medio de los Estados Unidos el propio Pérez Rosales y Vicuña Mackenna: individualismo, egoísmo, inhumanidad. Sin embargo, Pérez Rosales comprobaba, a partir de los cambios que observó en California, en qué sentido el destino de los países se definía dependiendo de la inclusión o marginación de dicho modelo de modernidad. A partir de lo anterior, el viajero sudamericano se atrevía a predecir el futuro de California:

California, olvidada, virgen e inculta, después de una guerra desastrosa [...] cayó en poder de los norteamericanos el año de 1847, y desde entonces desde el fondo del abatimiento y de la nada, escarnio de los partidos que la vendieron, y de cuantos contemplan impasibles los atrasos de las repúblicas hermanas, marcha con paso firme y gigantesco a la vanguardia de las de Occidente a quienes perderá pronto de vista en la carrera de la industria, de la fuerza y del progreso (Pérez Rosales, 2007, p. 127).

La trasformación de California tras su anexión a los Estados Unidos no hacía nada más que reafirmar en qué medida el Río Grande no conformaba tan sólo una frontera política. Y lo que era aún más grave: la distancia entre ambas porciones del Nuevo Mundo se tornaban cada vez mayores. Como en un espejo invertido, ambas Américas se veían desde el fondo de sus diferencias.

\section{Conclusiones}

El contexto histórico que rodeó el viaje de latinoamericanos, tanto a Europa como a los Estados Unidos, lo constituyó el proceso de formación - jurídica, económica, como cultural -, de las nuevas repúblicas latinoamericanas posterior al proceso de Emancipación de España. En este punto, el desplazamiento a los países que por entonces se tenían como los más avanzados resultó un aspecto fundamental de dicho fenómeno histórico. De allí que examinar la experiencia de los viajeros latinoamericanos en los Estados Unidos permita entender en qué medida no todo lo que se discutió por la centuria decimonónica se hizo al interior de las fronteras, ni circunscrita sólo a ellas. Ello expresa que, de ningún modo, las identidades se conformaron sólo en relación a sí mismas. Sin el factor alteridad, sin enfrentar las diferencias no hay la "comunidad imaginada" que ha destacado Benedic Anderson (1983). 
En este sentido, América Latina emergió en el siglo XIX a partir del momento en que se definió lo extraño, en un acto que a la vez que delimitó las fronteras, reforzó las peculiaridades que separaban lo propio de lo desemejante. ${ }^{12}$

Por otro lado, la figura misma del viajero durante el siglo XIX es en sí un elemento de importancia. El que se desplazó a Europa y a los Estados Unidos, el que dejó un registro de tales periplos en los periódicos, como en libros o en conferencias, es el mismo que asumió funciones gubernamentales o parlamentarias de primer orden. Estos viajeros son personajes que se mueven entre la República política y la República de las letras. Su empresa es a la vez literaria, como política. Desde este punto de vista, analizar las experiencias de viajes en figuras como Domingo Faustino Sarmiento, Benjamín Vicuña Mackenna, José Martí, Vicente Pérez Rosales (por citar sólo a los aquí analizados) da un marco de comprensión a sus políticas de inmigración, de educación, respecto de su relación con los grupos indígenas, así como de su hostilidad o cercanía con los Estados Unidos.

La experiencia de los viajeros latinoamericanos en los Estados Unidos, a pesar de sus diferencias en tipos de recorridos, duración del viaje, edades y formación intelectual, posee algunos elementos en común.

En primer lugar, muchos advierten que lo que distancia a América Hispana de la Sajona corresponde al tipo de instituciones que ambas poseen. Algunos viajeros, como Pérez Rosales, relacionan el tipo institucional con el componente étnico, otros como Sarmiento, más bien encontraban una respuesta en el funcionamiento mismo de dichas instituciones: la utilización de la libertad, más que la libertad en un sentido abstracto. Sin embargo, a uno y a otro, la observación de la vida cotidiana en los Estados Unidos les permitía evaluar, desde la praxis diaria, cómo era la república norteamericana.

En segundo lugar, los viajeros de ningún modo tomaron lo observado en el País del Norte como un modelo a imponerse ciegamente. Muy por el contrario: muchos lo discutieron, reflexionaron sobre su posible utilidad en sus países o, definitivamente, lo visualizaron como una enseñanza de lo que "no se debía de hacer".

En tercer lugar, los viajeros se percataron que el futuro, el porvenir de la civilización occidental estaba en los Estados Unidos. A muchos,

12 Respecto a la relación entre viaje e identidad ver LEED, 1991; DOLAN, 2000; SCHAFFER, 2001; MARTÍNEZ, 2001; MORGAN, 2001 y SANHUEZA, 2006. 
como a Pérez Rosales o Vicuña Mackenna, les irritó los costos de la vía norteamericana a la modernidad (deshumanización, egoísmo extremo, materialismo) y sin embargo se vieron obligados a reconocer que aquel era el único acceso al progreso. La experiencia de viajes de latinoamericanos en los Estados Unidos expresó la posición conflictiva de su elite con el mundo moderno: ¿En qué medida era posible aplicar dicho modelo en las jóvenes repúblicas hispanoamericanas? ¿Atentaba este modelo contra su identidad?

A partir de tales elementos - relación con el mundo moderno, búsqueda de modelos - se fue construyendo la ficción de un subcontinente latinoamericano en los viajeros latinoamericanos, ya desde mediados del siglo XIX. Sólo así se entiende en qué sentido asumieron una defensa de lo propio y exaltación de unos valores asociados (espiritualismo, afectividad, solidaridad) frente a lo que percibían como característico del país del norte (utilitarismo, materialismo, vulgaridad). Los viajeros, en este sentido percibieron, mucho antes que José Enrique Rodó lo canonizara en el "Ariel" (1900), el enfrentamiento entre dos formas de civilización. ${ }^{13}$ Por fines del siglo XIX, ante los sucesos de la guerra entre los Estados Unidos y Cuba en 1898, la sensibilidad anti-norteamericana se hizo aún más patente. Estados Unidos no se vio como el aliado protector para América Latina como pudo haberlo sido anteriormente al amparo de la propia doctrina Monroe. De esta manera los Estados Unidos se representaron como lo opuesto de América Latina. Rubén Darío, quien por primera vez utilizó la figura del Ariel versus el Calibán, escribió por 1898 (1938, p. 160-162):

$\mathrm{Y}$ yo he visto a esos yankees en sus abrumadoras ciudades de hierro y piedra [...] Colorados, pesados, groseros, van por sus calles empujándose y rozándose animalmente a la caza de un dollar. El ideal de esos calibanes está circunscrito a la bolsa y a la fábrica [...]. Cantan ¡Home, sweet home! Y su hogar es una cuenta corriente.

Lo observado y registrado del País del Norte desde la medianía del siglo XIX, enfrentados al expansionismo norteamericano, se veía confirmado en sus postrimerías.

${ }^{13}$ Algunos han asumido que la visión latinoamericana de los Estados Unidos tiene sus raíces a partir del siglo XX, en especial tras el Ariel de Enrique Rodó. Lo que el estudio de viajeros indica es justamente que la discusión sobre los Estados Unidos comienza ya a mediados del siglo XIX: Ver RINKE, 2004. 


\section{Referências}

ANDERSON, Benedic. Imagined Communities. Reflections on the Origin and Spread of Nationalism. London: Editorial Verso, 1983.

BEILHARZ, Edwin; LÓPEZ, Carlos (Ed.). We Were 49ers! Chilean Accounts of the California Gold Rush. Pasadena, California: Ward Ritchie Press, 1976. BRINTRUP, Lilianet. Viaje y escritura. Viajeros románticos chilenos. New York: Peter Lang, 1992.

BURUMA, Ian, y Avishai Margalit. Occidentalism. New York: The Penguin Press, 2004.

CAMACHO ROLDÁN, Salvador. Notas de viaje (Colombia y los Estados Unidos). Bogotá: Publicaciones del Banco de la República, 1973. Tomos I y II. CHAKRABARTY, Dipesh. Habitations of Modernity. Chicago: University of Chicago Press, 2002.

CHAKRABARTY, Dipesh. Provincialing Europe. Princeton: Princeton University Press, 2000.

CHUCHIAK IV, John. Intelectuals, indians, and the press: the politicitazion of Justo Sierra O'Really`s Journalism and Views on the Maya in the Unites States. In: FEY, Ingrid; RACINE, Karen. (Ed.). Strange Pilgrimages: Exile, travel, and national identity in Latin America, 1800-1990s. Wilmington, Scholarly Resources, 2000: 59-74.

COLOMBI, Beatriz. Viaje intelectual. Migraciones y desplazamientos en América Latina (1880-1915). Buenos Aires: Beatriz Viterbo: 2004.

CONNEL-SMITH, Gordon. Los Estados Unidos y América Latina. Estudio de las relaciones entre Estados Unidos y el resto del continente americano. México, D.F.: Fondo de Cultura Económica, 1974.

DARÍO, Rubén. El triunfo de Calibán. El Cojo Ilustrado de Caracas, (1 de octubre de 1898): 676. In: MAMPES, E.K. Escritos inéditos de Rubén Darío. New York: Instituto de las Españas, 1938. p. 160-162.

DENT, David. The legacy of the Monroe Doctrine: Areference guide to U.S. involvement in Latin America and the Caribbean. Westport: Greenwood Press, 1999.

DOLAN. Exploring European Frontiers. British Travellers in the Age of Enlightenment. New York, St. Martin's Press, Inc., 2000.

ELSNER, Jás; RUBIÉS, Joan-Paul. (Ed). Voyages and visions. Towards a cultural history of travel. London: Reaction Books, 1999.

ERRAZURIZ, Rebeca. Sarmiento y Martí en los EE.UU: Imaginarios de la Modernidad, Universum. Talca, v. 23, n. 1, p. 40-63, 2008.

ETTE, Ottmar Weltbewußtsein. Alexander von Humboldt und das unvollendete Projekt einer anderen Moderne. Weilerwist: Velbrück Wissenschaft, 2002.

FEY, Ingrid; RACINE, Karen. (Ed.). Strange Pilgrimages: Exile, travel, and national identity in Latin America, 1800-1990s. Wilmington: Scholarly Resources, 2000. 
GUERRA, François-Xavier. Inventando la nación Iberoamérica. Siglo XIX. México: Fondo de Cultura Económica, 2003.

GUERRERO, Yoachim Cristián. La misión de Vicuña Mackenna a los Estados Unidos, 1865-1866. In: Boletín de la Academia Chilena de Historia,. Santiago de Chile, año 53, n. 97, p. 35-86, 1986.

HARRIS, Gilberto. Emigración y políticas gubernamentales en Chile durante el siglo XIX. Valparaíso: Ediciones de la Universidad Católica de Valparaíso, 1996.

HARTOG, François. El espejo de Herodoto. México: Fondo de Cultura Económica, 2003. (1 $1^{\text {a }}$ ed., 1980).

HERNÁNDEZ, Roberto. Los chilenos en San Francisco de California. Valparaíso: Imprenta San Rafael, 1930. v. I y II.

KATRA, William. "Rereading Viajes: Race, Identity, and national Destinity", en: HALPERIN, T. Et. Alli. Sarmiento. Author of a Nation. Berkeley: University of California Press, 1994 73-100.

KATRA, William. Sarmiento en los Estados Unidos. In: SARMIENTO, D.F. Viajes por Europa, África y América. Santiago: Editorial Universitaria y ALLCA, 1997. p. 853-912.

LEED, Eric J. The mind of the traveller. From Gilgamesh to global tourism. New York: Basic Books, 1991.

LEGRAND, Joseph. Close encounters of empire: Writing the cultural history of U.S.-Latin American. Relations Duke: Duke University Press, 1998.

LÓPEZ, Carlos. Chilenos in California. A study of the 1850, 1852 and 1860 Censuses. San Francisco: R and E Research Associates, 1973.

LÓPEZ, Carlos. Episodios chilenos en California. Valparaíso: Ediciones Universidad de Valparaíso, 1975.

MÁRQUEZ, José Arnaldo. Recuerdos de un viaje a los Estados Unidos de la América del Norte. Lima.: Fondo Editorial Universidad Mayor de San Marcos, 2003 ( $1^{\text {a }}$ ed.: Lima: Imprenta del Comercio, 1862).

MARTÍ, José. En los Estados Unidos. México: ALLCA XX, 2003.

MARTÍNEZ, Frédéric. El nacionalismo cosmopolita. La referencia europea en la construcción nacional en Colombia. 1845-1900. Bogotá: Banco de la República; Instituto Francés de Estudios Andinos, 2001.

MICHAELSEN, Scout; JOHNSON, David E. Teoría de la frontera. Barcelona: Gedisa, 2003.

MIGNOLO, Walter. Local histories/global designs: coloniality, subaltern knowledges and border thinking. Princeton: Princeton University Press, 2000.

MIGNOLO, Walter. The Idea of Latin America. Malden: Blackwell Publishing, 2005.

MORGAN, Marjorie. National identities and travel. In: Victorian Britain. Houndmills: Palgrave, 2001. 
PÉREZ ROSALES, Vicente. Diario de un viaje a California. 1848-1849. In: LATORRE, G.; MARTÍNEZ, M.E. (Ed). Santiago de Chile: Tajamar, 2007. PÉREZ ROSALES. Vicente. Algo sobre California. Revista de Santiago. Santiago: Primera Época, v. 6, n. 3-10, p. 127-136, 185-193, 1850.

PÉREZ ROSALES, Vicente. Recuerdos del Pasado. Santiago de Chile: Gutemberg, 1886.

RACINE, Karen; DE MIRANDA, Francisco. A transatlantic life in the age of revolution. Wilmington: SR Books, 2003.

RAMA, Carlos. La imagen de los Estados Unidos en la América Latina. De Simón Bolívar a Salvador Allende. México: Secretaría de Educación Pública, 1975.

RINKE, Stefan. Begegnungen mit dem Yankee. Nordamerikanisierung und soziokultureller Wandel in Chile 1898-1990. Böhlau: Colonia, 2004.

SALVATORE, Ricardo. Culturas imperiales: experiencia y representación en América, África y Asia. Buenos Aires: Beatriz Viterbo, 2005.

SANHUEZA, Carlos. En busca de un lugar en el mundo. Viajeros latinoamericanos en la Europa del siglo XIX. Estudos Ibero-Americanos, v. XXXIII, n. 2, p. 51-75, 2007.

SANHUEZA, Carlos. Chilenos en Alemania y alemanes en Chile. Viaje y nación en el siglo XIX. Santiago de Chile: LOM-DIBAM, 2006.

SARMIENTO, Domingo Faustino. Viajes por Europa, África y América. Santiago: Editorial Universitaria y ALLCA, 1997.

SCHAFFER, Marguerite S. See America First: Tourism and National Identity, 1880-1940. Washington, D.C.: Smithsonian Institution Press, 2001.

SULLIVAN, Henry. The border that refused to go away: The Río Grande as replication of the Rhine-Danube frontier. In: GALOPPE, Raúl; WEINER, Richard. Explorations in subjectivity, borders, and demarcation: A fine line. Lanham/Boulder/NewYork/ Toronto/Oxford: University Press of America, Inc. 2005. p. 3-24.

SZURMUK, Mónica. Women in Argentina: early travel narratives. Gainesville: University Press of Florida, 2000.

VENN, Couza. Occidentalism. London: Sage, 2000.

VICUÑA MACKENNA, Benjamín. Diez meses de Misión a los Estados Unidos de Norteamérica como ajente confidencial de Chile. Santiago de Chile: Imprenta de la Libertad, 1867. Tomo I.

VICUÑA MACKENNA, Benjamín. Pájinas de mi Diario durante los tres años de viaje. 1853-54-55. Santiago de Chile: Imprenta del Ferrocarril, 1856.

VIÑAS, David. Viajeros argentinos a Estados Unidos. Parabellum: Buenos Aires, 2008.

WALLERSTEIN, Immanuel. The modern world system. New York: Academic Press, 1974-1989.

ZORAIDA, J.; MEYER, L. México frente a los Estados Unidos. México: Fondo de Cultura Económica, 1982. 